\title{
Experience with the Mitroflow aortic bioprosthesis
}

From October 1985 to May 1990, the Mitroflow bovine pericardial valve was placed in the aortic position in 168 patients (97 men, 71 women) with a mean age of 69.7 years. Eighty-nine patients had isolated aortic valve replacement, and 79 had aortic valve replacement and additional procedures. Follow-up over $7 \frac{1}{2}$ years includes 781 patient years $(\mathbf{4 2 6}$ for isolated aortic valve replacement). Mean follow-up time is 56 months. Peak-to-peak gradients (in millimeters of mercury) measured in the intraoperative period averaged $11.0 \pm 8.7,11.8 \pm 10.8$, and $8.6 \pm 8.2$ for $19 \mathrm{~mm}, 21 \mathrm{~mm}$, and $23 \mathrm{~mm}$ valves, respectively. Hospital mortality was $7.3 \%$ (14 patients); all deaths were non-valve related. Late mortality of $20.1 \%$ in 31 patients resulted from cardiac failure $(n=8)$, sepsis $(n=4)$, valve reoperation $(n=1)$, non-cardiac causes $(n=15)$ and sudden, unknown causes $(n=3)$. Fifteen thromboembolic episodes occurred, but only three late thromboembolic episodes occurred in isolated aortic valve replacement without other risk factors. Four early and four late episodes of endocarditis occurred. Seven patients had clinical valve dysfunction, and five others required reoperation for structural deterioration, with one death. At 94 months, overall survival was $64 \% \pm 5 \%$. Freedom from thromboembolic episode was $87 \% \pm 3 \%$ and $90 \% \pm 4 \%$ for isolated aortic valve replacement. Freedom from combined reoperation or clinical dysfunction was $75 \% \pm 8 \%: 64 \% \pm 15 \%$ for those under 70 years of age, and $87 \% \pm 7 \%$ for those 70 years of age and older. The valve has favorable hemodynamics. Durability begins to decline during the sixth year after implantation, possibly at a slower rate in patients older than 70 years of age. (J Thorac CardiovasC Surg 1994;108:215-20)

Richard A. Moggio, MD, Richard W. Pooley, MD, Mohan R. Sarabu, MD, Joseph Christiana, BS, Allan W. Ho, BS, MPH, and George E. Reed, MD, Valhalla, N.Y.

$\mathrm{D}$ sign and manufacturing of bioprosthetic valves have undergone considerable change over 30 years of development. Fresh autologous pericardium and fascia lata, mounted on rigid trileaflet metal stents, provided an alternative to mechanical prostheses, especially in older patients and those unable to tolerate anticoagulants. ${ }^{1,2}$ The development of glutaraldehyde tissue fixation helped increase the durability and commercial availability of bioprostheses. The Ionescu-Shiley bovine pericardial prosthesis (Shiley, Inc., Irvine, Calif.) produced excellent hemodynamics but had significant deterioration between 5 and 10 years after implantation. ${ }^{3,4}$ The second generation of pericardial valves used different mounting tech-

From the Westchester County Medical Center, New York Medical College, Valhalla, N.Y.

Received for publication June 17, 1993.

Accepted for publication Dec. 14, 1993.

Address for reprints: Richard A. Moggio, MD, Cardiothoracic Surgery, Westchester County Medical Center, New York Medical College, Valhalla, NY 10595.

Copyright 1994 by Mosby-Year Book, Inc.

$0022-5223 / 94 \$ 3.00+0 \quad 12 / 1 / 53692$ niques and flexible low profile stents, but abrasion between tissue and cloth covering remains a likely cause of valve failure. ${ }^{5}$ The Mitroflow bovine pericardial valve (Mitroflow International, Inc., Richmond, British Columbia, Canada) was introduced in 1982 and approved for investigational study in the United States in 1985. The valve was used at the Westchester County Medical Center from October 1985 to May 1990 in the aortic position in 168 patients and is the basis for this report.

\section{Patients and methods}

Clinical material. At the Westchester County Medical Center, 97 men and 71 women underwent placement of the Mitroflow valve in the aortic position. Ages ranged from 17 to 85 years with a mean age of 69.7 years and a median age of 70.0 years. Fourteen patients had previously undergone cardiac operation. Seven patients were in atrial fibrillation at the time of operation. The primary valvular diagnosis was aortic stenosis in 86 patients, aortic insufficiency in 25 , a mixed lesion in 55 , and prosthetic malfunction in two. Preoperative New York Heart Association classifications included five patients in class I, 42 in class II, 92 in class III, and 29 in class IV.

The Mitroflow investigational study was approved by the Investigation Review Committee of the Westchester County 


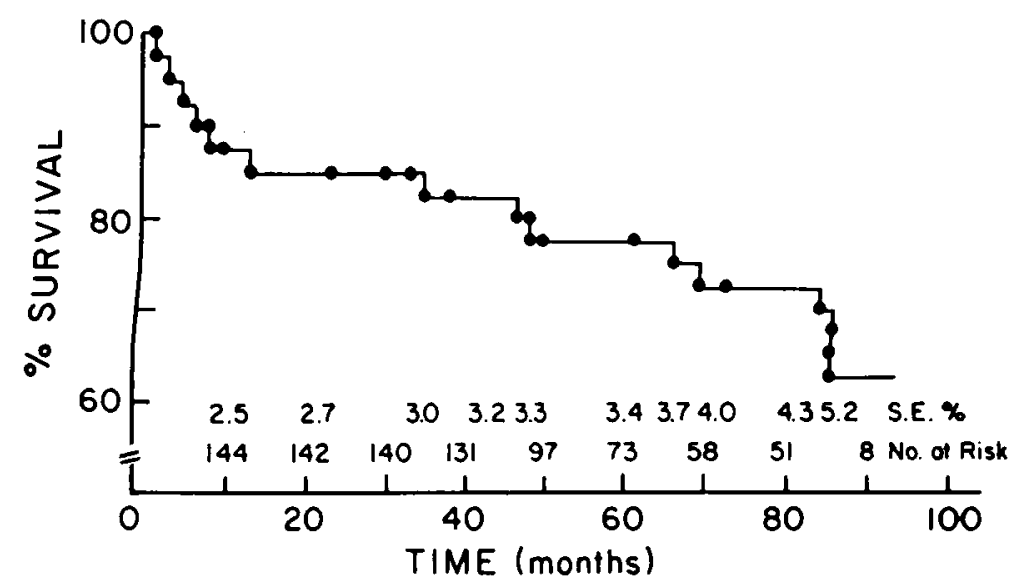

Fig. 1. Survival curve for all patients undergoing AVR. S.E., Standard error.

Table I. Peak-to-peak gradients measured in the intraoperative period across the aortic prostheses at time of implantation

\begin{tabular}{ccccc}
\hline $\begin{array}{c}\text { Valve size } \\
(\mathrm{mm})\end{array}$ & No. of patients & $\begin{array}{c}\text { Peak-to-peak } \\
\text { gradient }(\mathrm{mm} \mathrm{Hg})^{*}\end{array}$ & Range & $\begin{array}{c}\text { Cardiac output } \\
(\text { L/min })^{*}\end{array}$ \\
\hline 19 & 14 & $11.0 \pm 8.7$ & $0-25$ & $4.1 \pm 0.9$ \\
21 & 39 & $11.8 \pm 10.8$ & $0-40$ & $4.7 \pm 1.1$ \\
23 & 36 & $8.6 \pm 8.2$ & $0-35$ & $5.3 \pm 1.0$ \\
25 & 27 & $5.2 \pm 5.2$ & $0-15$ & $5.3 \pm 1.3$ \\
27 & 6 & $1.7 \pm 4.1$ & $0-10$ & $5.7 \pm 0.9$ \\
29 & 1 & 0 & 0 & 6.4
\end{tabular}

*Values expressed as mean \pm standard deviation.

Medical Center and by the Office of Research of the New York Medical College. Consent to use the Mitroflow valve was obtained individually from the patient by the operating surgeon.

\section{Results}

Operative results and mortality. Operation was performed with standard techniques of cardiopulmonary bypass, systemic hypothermia, and cold blood hyperkalemic cardioplegic solution. The aortic bioprosthesis was placed in the supraannular position with mattress sutures. Isolated aortic valve replacement (AVR) was performed in 89 patients, 50 men and 39 women. Thirteen patients underwent concomitant mitral valve repair or replacement. Sixty-four patients underwent additional coronary artery bypass procedures. The most commonly used valves were 21 and $23 \mathrm{~mm}$, and 124 of the 168 patients had either 19,21, or $23 \mathrm{~mm}$ valves placed.

Whenever possible, gradients were measured across the aortic valves and the cardiac output was recorded. These measurements were obtained by use of 20 -gauge needles and simultaneous left ventricular and aortic root recordings. These values are in Table I. An average peak-to-peak gradient of $11 \mathrm{~mm} \mathrm{Hg}$ was measured across
14 of the $19 \mathrm{~mm}$ valves, with an average peak gradient of $12 \mathrm{~mm} \mathrm{Hg}$ across 39 of the $21 \mathrm{~mm}$ valves and an average peak gradient of $9 \mathrm{~mm} \mathrm{Hg}$ across 36 of the $23 \mathrm{~mm}$ valves.

Fourteen patients died before leaving the hospital, eight within the first 30 days and six up to 6 months after the operation. Three of 14 had isolated AVR (3.3\%), and 11 underwent additional procedures (13.9\%). Most patients died of combined multisystem failure and ventricular failure. None died of primary valve failure.

Follow-up and analysis. All information has been compiled for Food and Drug Administration and Mitroflow International, Inc., review on patient report forms. Follow-up at 6 months to 1 year was accomplished through direct patient examination, questionnaires to patients or following physicians, and telephone contact with patients, families, and physicians. Two of 168 patients were lost to follow-up at periods of 6 months to 2 years after discharge from the hospital. Accumulated follow-up is 781.1 patient-years with a mean time of 55.8 months (425.8 years, mean 57.4 months, for isolated AVR). Descriptive statistics were derived with the SPSS software (SPSS, Inc., Chicago, Ill.), and the life-table 


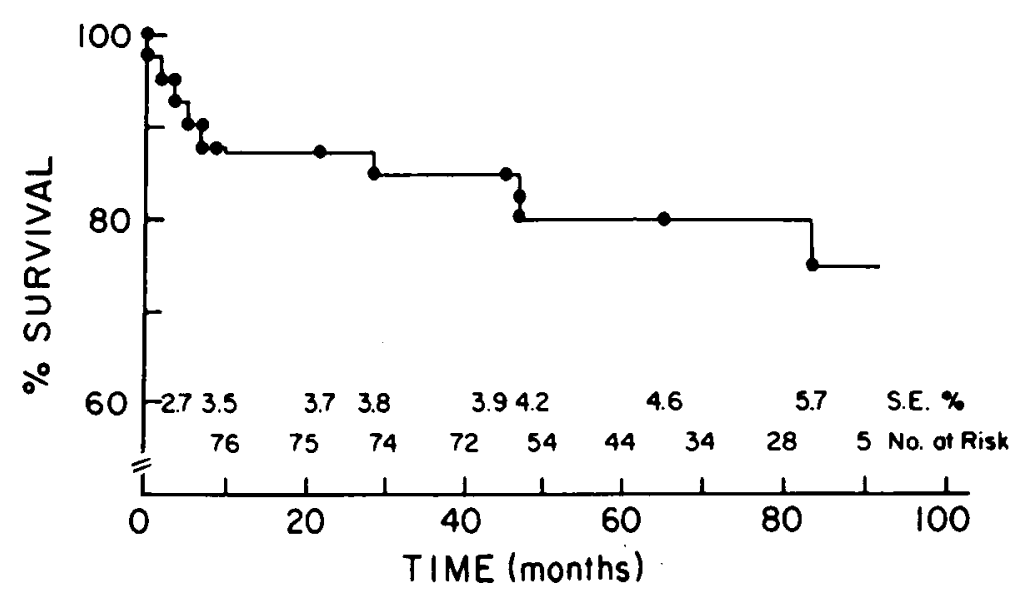

Fig. 2. Survival curve of 79 patients undergoing isolated AVR. S.E., Standard error.

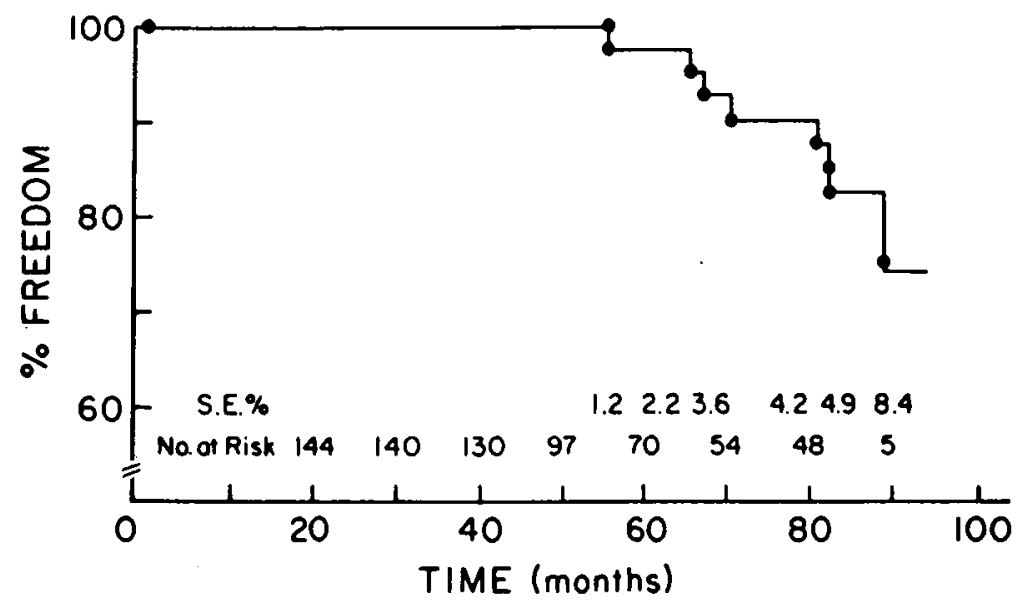

Fig. 3. Freedom from valve deterioration for all patients up to 94 months after AVR. S.E. Standard error.

analysis and survival curves were derived from the product-limit method (Kaplan-Meier) with the BMDP statistical software (BMDP Statistical Software, Los Angeles, Calif.).

Late results and mortality. Of the 154 patients discharged from the hospital, 31 died from 6 months to 85 months after operation. Among twelve cases of isolated valve replacements, three patients died suddenly at 6 months, two died of heart failure at 6 months, two of ventricular failure, and four of non-cardiac causes. One died after reoperation to replace a deteriorated Mitroflow aortic valve (described later). Among patients who had undergone reoperation or combined valve and bypass grafting, 19 late deaths occurred. One patient died of persistent endocarditis, six died of congestive heart failure as a result of ventricular dysfunction, 11 died of non-cardiac causes, and one died after reoperation to repair a mitral paravalvular leak.
Twenty of 31 late deaths involved patients 70 years of age or older at operation. Fifteen of these 20 patients died of non-cardiac causes, and none died of valve-related causes. The overall survival curve (Fig. 1) shows late survival at $7 \frac{1}{2}$ years of $64 \% \pm 5 \%$. Survival of those with isolated AVR is $75 \% \pm 6 \%$ at 94 months (Fig. 2). At follow-up of 145 patients, 130 were in New York Heart Association class I, 12 in class II, two in class III, and one in class IV.

Valve-related complications

Valve failure. Five patients required reoperation for failure of the bioprosthesis from 55 to 89 months after implantation (mean 68 months). Ages at primary operation for three men and two women ranged from 39 to 73 years. Three valves had calcification and leaflet tears along the struts, and two were calcified and stenotic without tears. No pattern was apparent with regard to valve size, gradient, or gender at time of deterioration or mode 


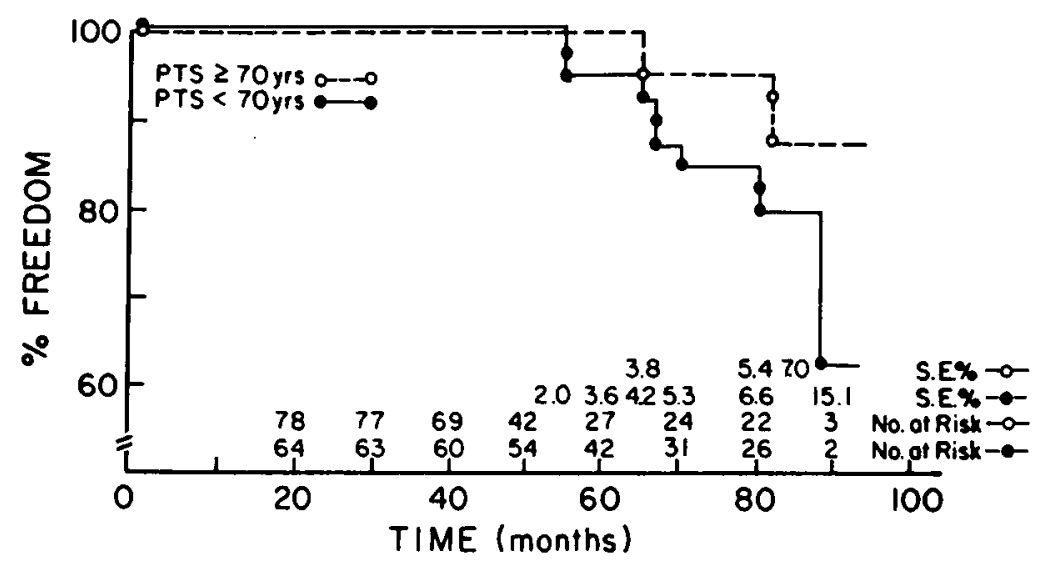

Fig. 4. Freedom from valve deterioration up to 94 months after aortic valve replacement, by age group. Open circle (O), Patients 70 years of age or older at implantation; closed circle (O), patients younger than 70 years of age at implantation. S.E., Standard error.

of valve failure. Freedom from reoperation for all patients was $87 \% \pm 7 \%$ at 94 months.

Seven patients with prosthetic dysfunction evidenced by aortic regurgitation were followed-up by clinical examination or echocardiography. Ages at operation ranged from 55 to 72 years in five men and two women. Clinical regurgitation was noted from 68 to 82 months after the operation, with a mean time of 76 months. One patient died after unrelated cardiac operation. The other six did not show clinical deterioration. Freedom from clinical valve dysfunction was $88 \% \pm 4 \%$. The combined freedom from either reoperation or clinical regurgitation is shown in Fig. 3: $75 \% \pm 8 \%$ at 94 months. The combined freedom for those under 70 years of age at implantation is $64 \% \pm 15 \%$, and that for those 70 years of age or greater is $87 \% \pm 7 \%$ (Fig. 4).

Endocarditis. Endocarditis was found in eight patients. Four of these cases occurred in the first 6 months after operation and were associated with prolonged hospitalizations with multiple complications including blood cultures positive for sepsis. None of these cases involved primary endocarditis.

Four remaining patients had late endocarditis. These occurred from 19 to 41 months after the operation and involved enterococcus and staphylococcus organisms. No valve developed clinical regurgitation. Freedom from endocarditis at 90 months was $94 \% \pm 29 \%$.

Anticoagulation and related hemorrhage. At the time of discharge from the hospital, 110 patients received warfarin sodium (Coumadin), consistent with the surgeons' policy, in general, to maintain anticoagulation for 3 months in those able to tolerate the medication. In addition, 11 patients received warfarin and dipyridamole (Persantine), whereas 20 others received aspirin or aspi- rin and dipyridamole therapy. The remainder had no anticoagulation or antiplatelet medication.

Three patients had significant hemorrhage while receiving warfarin; no hemorrhaging was fatal. The medication was discontinued in all patients.

Thromboembolic events. Fifteen patients had thromboembolic events, from 3 days to 78 months after the operation. Three of these events occurred in the early postoperative period, and only one of the patients was receiving anticoagulants at the time. Among 12 patients with late embolic events, four were receiving warfarin, three were receiving aspirin, dipyridamole, or both, and five were receiving no anticoagulation or antiplatelet medication. All four patients with late embolic events who were receiving warfarin had associated mitral valve disease with atrial fibrillation. Late thromboembolism occurred in three patients with isolated AVR and no other associated risk factor, two of whom were receiving aspirin at the time of the event. Freedom from thromboembolism as shown in Fig. 5 was $87 \% \pm 3 \%$ at $71 / 2$ years, whereas freedom from thromboembolism with isolated AVR was $90 \% \pm 4 \%$.

Table II shows the linearized event rates for the major complications of AVR. The total cumulative follow-time was 781.1 years for all patients and 425.8 years for isolated AVR, as of August 1993.

\section{Discussion}

The design of the Mitroflow pericardial valve incorporated a single piece of pericardium applied to the outside of a flexible Delrin stent (DuPont Corp., Wilmington, Del.) to maximize valve orifice area. Stent commissure sutures were placed on the outside surface to minimize stress at this point. The low transvalvular peak-to-peak 


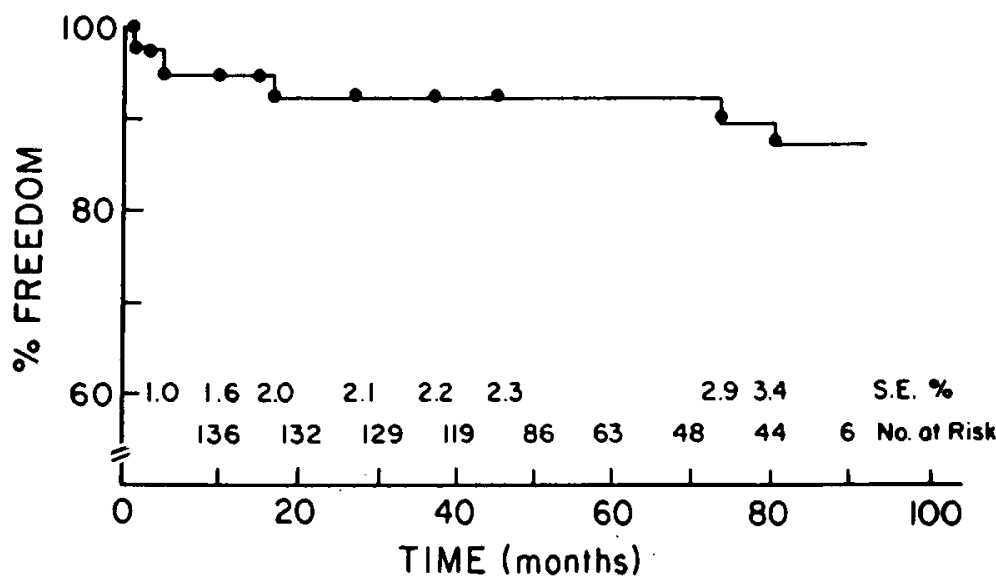

Fig. 5. Freedom from thromboembolism for all patients up to 92 months after AVR. S.E., Standard error.

gradients measured at operation indicated favorable hemodynamics and made the valve a well suited prosthesis in smaller sizes. The gradient across the valves compared favorably to those measured by Revuelta, Garcia-Rinaldi, and Duran ${ }^{6}$ in follow-up of the IonescuShiley valves and by Pelletier and associates ${ }^{7}$ across the Carpentier-Edwards pericardial valve (Baxter Healthcare Corp., Edwards Division, Santa Ana, Calif.) measured in the postoperative period. Cosgrove and associates $^{8}$ measured peak-to-peak gradients in the intraoperative period and found mean gradients of 24.3 $\mathrm{mm} \mathrm{Hg}$ and $15.3 \mathrm{~mm} \mathrm{Hg}$ for the 21 and $23 \mathrm{~mm}$ Carpentier-Edwards pericardial valve, respectively. Perier and associates ${ }^{9}$ measured postoperative mean gradients of 16 , 15.3, and $11.9 \mathrm{~mm} \mathrm{Hg}$ across 19,21 , and $23 \mathrm{~mm}$ valves, respectively. Our peak gradients of 11.8 and $8.6 \mathrm{~mm} \mathrm{Hg}$ for the same sized Mitroflow valves seem to indicate improved hemodynamics with the Mitroflow valve. However, Cosgrove and associates ${ }^{8}$ pointed out the importance of using multiple hemodynamic measurements to fully characterize the performance of any given valve. Further, intraoperative measurements are difficult to compare because different techniques may be used. These results cannot be closely correlated with those obtained by echocardiography and cardiac catheterization in the postoperative period because of these differences in techniques and the role of patient-related and prosthesis-related factors.

Endocarditis, thromboembolism, and durability remain critical determinants of prosthetic function.

Patient-related factors are likely to be more important than valve factors in determining the incidence of endocarditis. Our linearized rate of 1.02/100 patientyears is within the range reported by Jamieson ${ }^{10}(0.6 \%)$ patient-year) and Loisance ${ }^{11}$ and their associates for the
Table II. Linearized event rates for all patients and for patients with isolated aortic valve replacement per 100 patient-years

\begin{tabular}{lcc} 
& All & \\
& patients & Isolated AVR \\
\hline Hemorrhage & 0.38 & 0.23 \\
Endocarditis & 1.02 & 1.17 \\
Thromboembolism & 1.92 & 0.94 \\
Thromboembolism and & 0.90 & 0.70 \\
$\quad$ no other risk factor & & \\
Clinical valve dysfunction & 0.90 & 0.94 \\
Reoperation for failed valve & 0.64 & 0.47 \\
\hline
\end{tabular}

Mitroflow valve, and by Gonzalez-Lavin and associates ${ }^{4}$ $(1.9 \%$ /patient-year) for the Ionescu-Shiley valve. Pelletier and associates ${ }^{12}$ showed a higher rate of endocarditis with pericardial than with porcine aortic bioprostheses (1.45\%/patient-year versus $0.56 \%$ /patient-year), but freedom-rate curves for AVR were not significantly different.

Thromboembolism rates are influenced not only by valve design but also by clinical factors such as anticoagulants, atrial fibrillation, mitral valve disease, and vascular sources of emboli. Seven of the twelve late embolic events in our series occurred in patients with other predisposing conditions. Jamieson and associates ${ }^{10}$ reported a high rate of thromboembolism with the Mitroflow valve (7.0\%/patient-year), although Loisance and associates ${ }^{11}$ reported a low incidence of events $\left(0.5 \%\right.$ /patient-year). Pelletier and associates ${ }^{12}$ reported a higher rate for pericardial valves than for porcine aortic valves ( $1.36 \%$ /patient-year versus $0.56 \%$ /patient-year), but, again, actuarial curves of freedom were not significantly different. 
The central concern of durability cannot be fully addressed at a follow-up of $71 / 2$ years. The comparison by Pelletier and associates ${ }^{12}$ of porcine and pericardial valves indicated a divergence in freedom from valve failure at 6 years. This failure was more pronounced in mitral than aortic valve replacements, and more failures occurred with the Ionescu-Shiley prosthesis compared with the Mitroflow or Carpentier-Edwards valves.

Gonzalez-Lavin ${ }^{4}$ and Masters ${ }^{13}$ and their associates showed significant increases in failure rates between 6 and 10 years after implantation of both standard and lowprofile Ionescu prostheses. At 10 years, however, the freedom from failure in patients older than 60 years of age $(66 \% \pm 10 \%)$ was significantly better than that for patients between 40 and 60 years of age $(41 \% \pm 9 \%)$. Loisance and associates ${ }^{11}$ reported freedom from reoperation for aortic Mitroflow valves declining from $93.4 \% \pm 2.6 \%$ at 5 years to $55.1 \% \pm 10 \%$ at 8.5 years. Age groups were not distinguished in this report. The decline in durability in our series during the sixth year is similar to that shown by Loisance and associates. ${ }^{11}$ Older patients in our group appear to have longer valve durability than do younger ones: only one quarter of patients with valve deterioration were 70 years of age or older at the time of initial operation, but these older patients made up more than half of our series.

The favorable hemodynamics of the Mitroflow aortic prosthesis combined with the trend toward longer durability in the elderly continue to make the bovine pericardial prosthesis a satisfactory valve for implantation in the older age group. In patients younger than 70 years of age, a decline in durability between the sixth and eighth year may narrow the indications for implantation in this group.

\section{REFERENCES}

1. Ionescu MI, Ross DN. Heart valve replacement with autologous fascia lata. Lancet 1969;2:335-9.

2. Moggio RA, Reed GE. Long-term follow-up of autologous fascia lata valves. In: Gabbay S, Cabrol C, eds. Calcification and degeneration of heart valve prostheses. Paris: Communication Medicale, 1987;109-17.

3. Walley VM, Keon WJ. Patterns of failure in Ionescu-Shiley bovine pericardial bioprosthetic valves. J THORAC CARDIOVASC SURG 1987;93:925-33.

4. Gonzalez-Lavin L, Gonzalez-Lavin J, Chi S, Lewis B, Amini S, Graf D. The pericardial heart valve in the aortic position ten years later. J THORAC CaRdiovasc SuRG 1991;101:75-80.

5. Wheatley DJ, Fisher J, Reece IJ, Spyt T, Breeze P. Primary tissue failure in pericardial heart valves. $J$ THORAC CARDIOVASC SURG 1987;94:367-74.

6. Revuelta JM, Garcia-Rinaldi JL, Duran CG. The IonescuShiley pericardial valve in the small aortic annulus: A 7-year experience. In: Bodnar E, Yacoub M, eds: Biologic and bioprosthetic valves. New York: Yorke Medical Books, 1986:226-32.

7. Pelletier LC, Leclerc Y, Bonan R, Crepeau J, Dyrda I. Aortic valve replacement with the Carpentier-Edwards pericardial bioprosthesis: clinical and hemodynamic results. J Cardiac Surg Supp 1988;3:405-12.

8. Cosgrove DM, Lytle BW, Gill CC, et al. In vivo hemodynamics comparison of porcine and pericardial valves. $\mathrm{J}$ Thorac Cardiovasc Surg 1985;89:358-68.

9. Perier P, Mihaileann S, Fabiani JN, et al. Long-term evaluation of the Carpentier-Edwards valve in the aortic position. J Cardiac Surg 1991;6(Suppl):589-94.

10. Jamieson WRE, Gercin AN, Ling H, Miyagishima T, Janusz MT, Tyers GFO. The Mitral Medical pericardial bioprosthesis: new generation bovine pericardial prosthesis. J Cardiac Surg Supp 1988;3:413-28.

11. Loisance DY, Mazzocotelli JP, Bertrand PC, Deleuze PH, Cachera JP. Mitroflow pericardial valve: long-term durability. Ann Thorac Surg 1993;56:131-6.

12. Pelletier LC, Carrier M, Leclerc Y, Lepage G, deGuise P, Dyrda. I. Porcine versus pericardial bioprosthesis: a comparison of late results in 1593 patients. Ann Thorac Surg 1989;47:352-61.

13. Masters RG, Pipe AL, Bedard JP et al. Long-term clinical results with the Ionescu-Shiley pericardial xenograft. J ThORAC Cardiovasc Surg 1991;101:81-9. 